\title{
国 \\ Paradoxical Cerebral Embolization \\ Report Caused by Thrombus-In-Transit via a Patent Foramen Ovale in a Patient with Symptomatic Pulmonary Embolism: A Case Report
}

Hiroki Suenaga, Junya Tsurukiri, Takahisa Kato, Kyosuke Matsunaga, Naoko Ogawa, Akito Oiwa, Naruaki Otake, and Jushi Numata

Objective: Acute pulmonary embolism (PE) is a life-threatening cardiovascular event associated with high mortality and morbidity. The presence of a patent foramen ovale (PFO) in patients with acute PE represents a risk factor for mortality. Furthermore, a thrombus-in-transit via a PFO with impending paradoxical embolism carries a high mortality rate.

Case Presentation: An adult patient with ischemic stroke caused by paradoxical embolism following PE underwent mechanical thrombectomy and achieved successful recanalization. Initial CT pulmonary angiography (CTPA) showed not only pulmonary thromboemboli but also bilateral atrial thromboemboli. During hospitalization, transesophageal echocardiography (TEE) revealed the PFO with a right-to-left shunt. Two months after rehabilitation undergone by the patient, PE completely disappeared and PFO closure was conducted to reduce the recurrence risk of ischemic stroke.

Conclusion: Not only cardiologists but also interventional neurologists should understand that CTPA can demonstrate the thrombus-in-transit through the PFO and provides a reliable prediction of the sudden onset of ischemic stroke in patients with symptomatic PE. When identified, considering a case-by-case treatment approach by multidisciplinary teams is essential for preventing further life-threatening paradoxical embolization.

Keywords > cryptogenic stroke, pulmonary embolism, thrombus-in-transit, paradoxical embolization

\section{Introduction}

Acute pulmonary embolism (PE) is a life-threatening cardiovascular event associated with high mortality and morbidity. Rapid diagnosis is essential, and CT pulmonary angiography (CTPA) allows not only an adequate visualization of pulmonary thromboemboli but also of right ventricular enlargement, which indicates right

$\overline{\text { Emergency and Critical Care Medicine, Tokyo Medical University }}$ Hachioji Medical Center, Hachioji, Tokyo, Japan

Received: January 14, 2021; Accepted: February 15, 2021 Corresponding author: Junya Tsurukiri. Emergency and Critical Care Medicine, Tokyo Medical University Hachioji Medical Center, 1163, Tatemachi, Hachioji, Tokyo 193-0998, Japan Email: junya99@tokyo-med.ac.jp

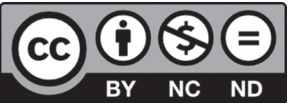

This work is licensed under a Creative Commons Attribution-NonCommercialNoDerivatives International License.

(C)2021 The Japanese Society for Neuroendovascular Therapy ventricular dysfunction. ${ }^{1)}$ The presence of a patent foramen ovale (PFO) in PE is associated with an increased risk of complications, especially ischemic stroke. ${ }^{2)}$ Furthermore, a thrombus-in-transit via a PFO with impending paradoxical embolism carries a high mortality rate. ${ }^{3,4)} \mathrm{We}$ herein report a case of acute ischemic stroke in a patient with acute symptomatic PE and PFO who underwent mechanical thrombectomy.

\section{Case Presentation}

Ten days before admission, a 55-year-old man was transferred to a local hospital because of a fall caused by syncope. The patient had experienced breathlessness and dyspnea one month before. He had a medical history of bipolar disorder, dyslipidemia, and alcohol-related liver disease. While the X-ray revealed a right tibiofibular fracture, MRI of the brain revealed no abnormalities. Transthoracic echocardiography (TTE) revealed an enlarged right atrium with a reduced left atrial size. Furthermore, his D-dimer levels were elevated to $15.8 \mu \mathrm{g} / \mathrm{mL}$ 

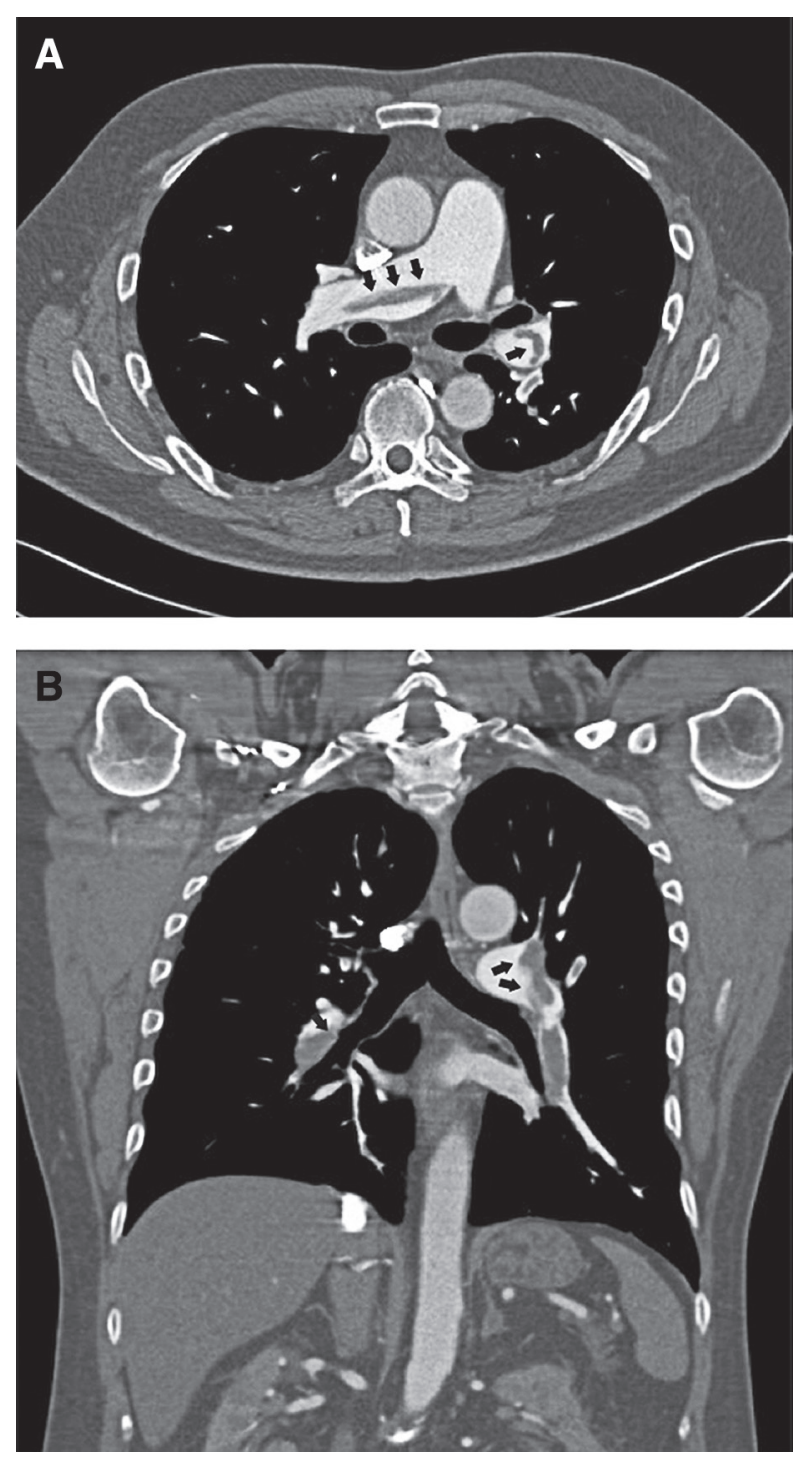

Fig. 1 CTPA revealed a thrombus involving multiple central, lobar, and segmental PAs (arrows). (A) axial image and (B) coronal image. CTPA: CT pulmonary angiography; PAs: pulmonary arteries

(reference range $[\mathrm{RR}],<0.30 \mu \mathrm{g} / \mathrm{mL}$ ). CTPA was subsequently performed, which revealed a thrombus involving multiple central, lobar, segmental pulmonary arteries (PAs) (Fig. 1). Ultrasound of the lower extremities revealed no deep venous thrombi. The patient was admitted to the hospital, and unfractionated heparin (UFH) and a direct oral anticoagulant (edoxavan, LIXIANA; Daiichi-Sankyo, Tokyo, Japan) were administered. Ten days after admission, consciousness disorder, aphasia, and right hemiparalysis suddenly developed and MR diffusion-weighted imaging of the brain revealed a high-signal intensity area in the middle cerebral artery (MCA). MR angiography revealed the absence of the left MCA, indicating an acute ischemic stroke (Fig. 2). Furthermore, the
Alberta stroke program early CT score was 7. The patient was then transferred to our emergency center for acute stroke care, including mechanical thrombectomy.

Physical examination on arrival at our emergency center revealed the following: Glasgow Coma Scale score, E4V4M5; blood pressure, 130/65 mmHg; heart rate, 60 beats/min; respiratory rate, 23 breaths/min; body temperature, $37.5^{\circ} \mathrm{C}$; and pupil diameter, $4 \mathrm{~mm}$. The National Institutes of Health Stroke Scale score was 22 points. Electrocardiography revealed a sinus rhythm. Subsequently, the patient underwent thrombectomy in the angiography suite.

\section{Neuroendovascular treatment}

Under local anesthesia with sedation, a 9 Fr long sheath was inserted into the right femoral artery and a 9 Fr. Optimo EPD (Tokai Medical Products, Aichi, Japan) was guided into the left internal carotid artery. Angiography demonstrated the left carotid T occlusion and the occlusion of the distal left MCA (Fig. 3A). A $200 \mathrm{~cm}$ Chikai 14 guide wire (Asahi Intecc, Aichi, Japan), Marksman catheter (Medtronic, Minneapolis, MN, USA), and ACE68 reperfusion catheter (Penumbra, Alameda, CA, USA) were coaxially inserted. The Marksman was guided to an area distal to the M2 segment of the left MCA. An EmboTrap II (Cerenovus, Irvine, CA, USA) was deployed through the Marksman, and mechanical thrombectomy was performed. Two passes led to recanalization with a grade 3 modified thrombolysis in cerebral infarction (Fig. 3B and C).

\section{Postoperative course}

UFH was initiated after achieving mechanical thrombectomy. We reviewed the initial CT images performed at the previous hospital and determined the thrombi was not only in the bilateral PA but also in bilateral atria (Fig. 4). The symptoms became markedly reduced, and anticoagulant therapy was switched from UFH to apixaban (ELIQUIS; Bristol Myers Squibb, New York, NY, USA and Pfizer, New York, NY, USA) on day 7 after the procedure. Transesophageal echocardiography (TEE) revealed a PFO $3.7 \mathrm{~mm}$ in size (Fig. 5). There were no thrombi, vegetation, or abnormal structures in the chamber, and a mobile plaque at the aorta was not observed. On day 25 after the procedure, CTPA revealed the absence of the thrombus in the main PA. The patient was discharged from our hospital for rehabilitation 28 days after admission and a modified Rankin Scale score of 2. Two months after rehabilitation, PE had completely disappeared and PFO closure was conducted to reduce the risk of recurrence of ischemic stroke. 


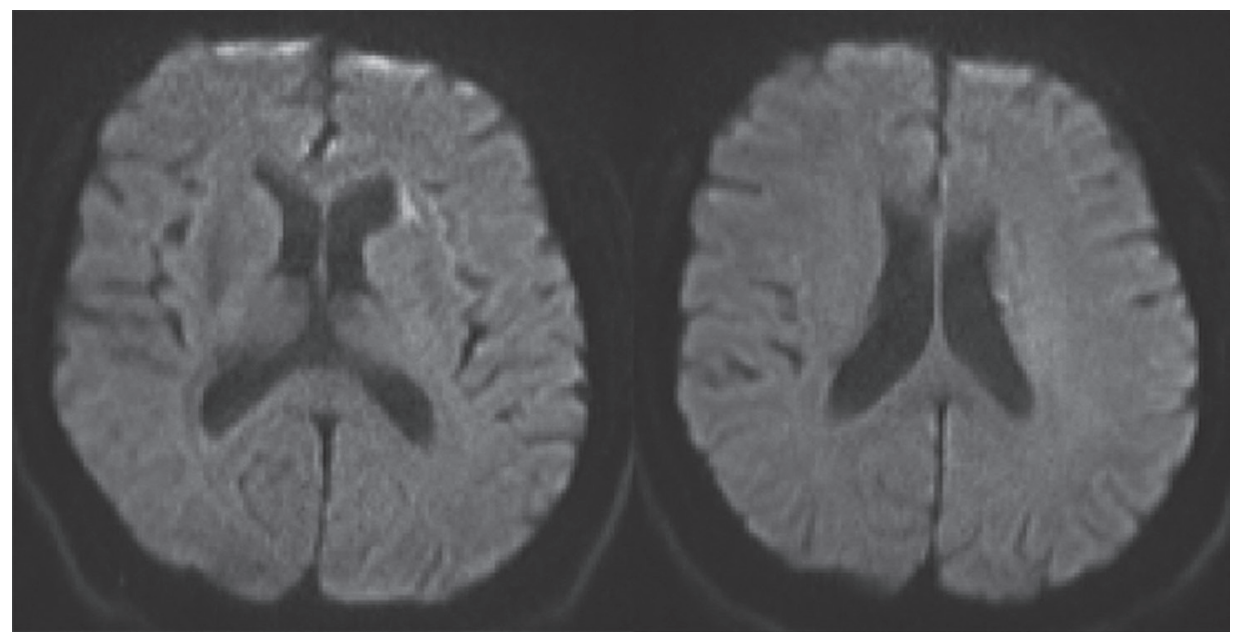

Fig. 2 MR diffusion-weighted imaging of the brain. Alberta Stroke Program Early CT score was 7.
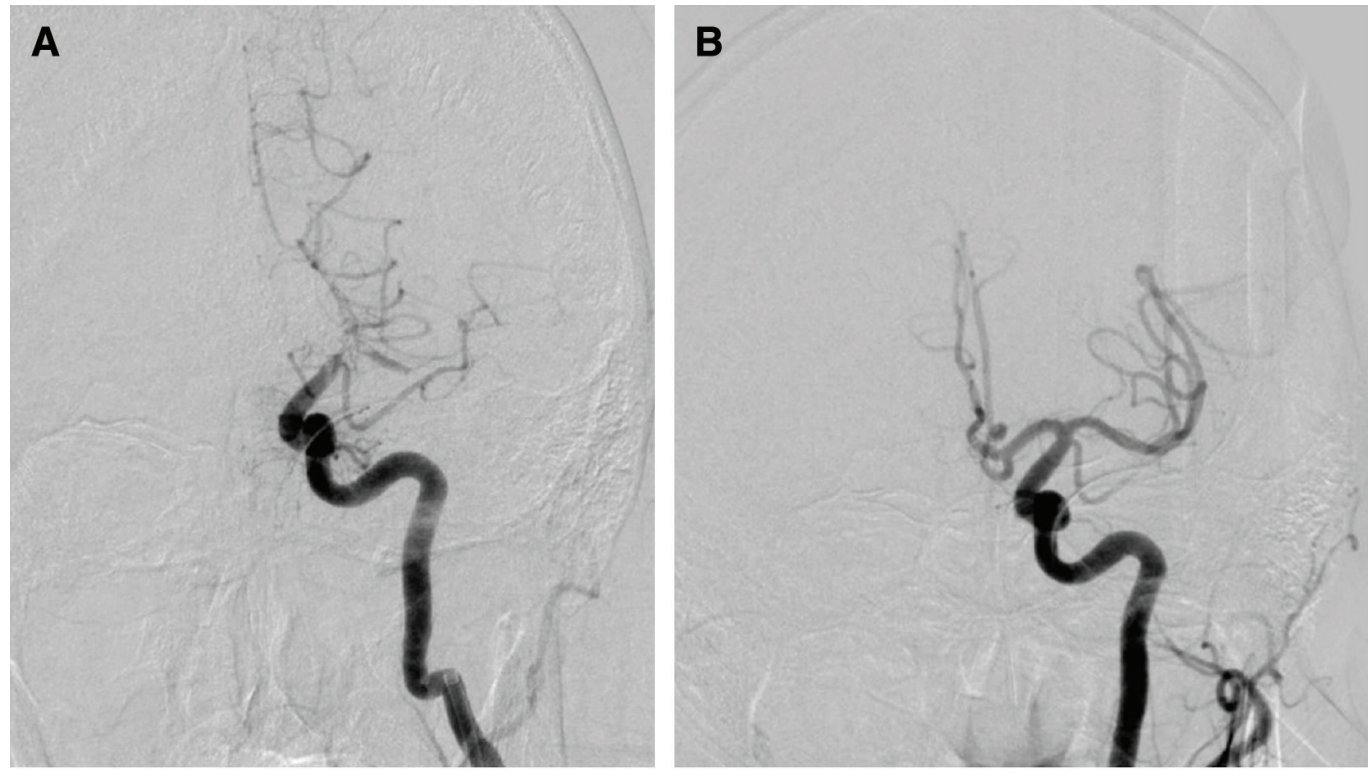

C
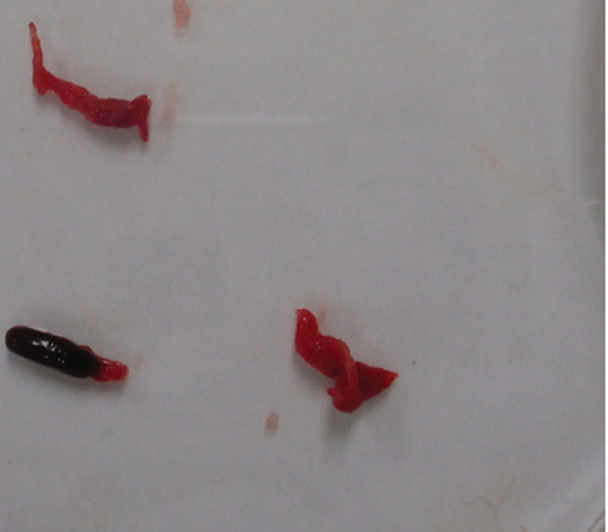

Fig. 3 Cerebral angiography. (A) Before mechanical thrombectomy, an angiography demonstrated the left carotid T occlusion and the occlusion of the distal left middle carotid artery. (B) After mechanical thrombectomy, two passes led to complete recanalization. (C) The thrombus were retrieved. 

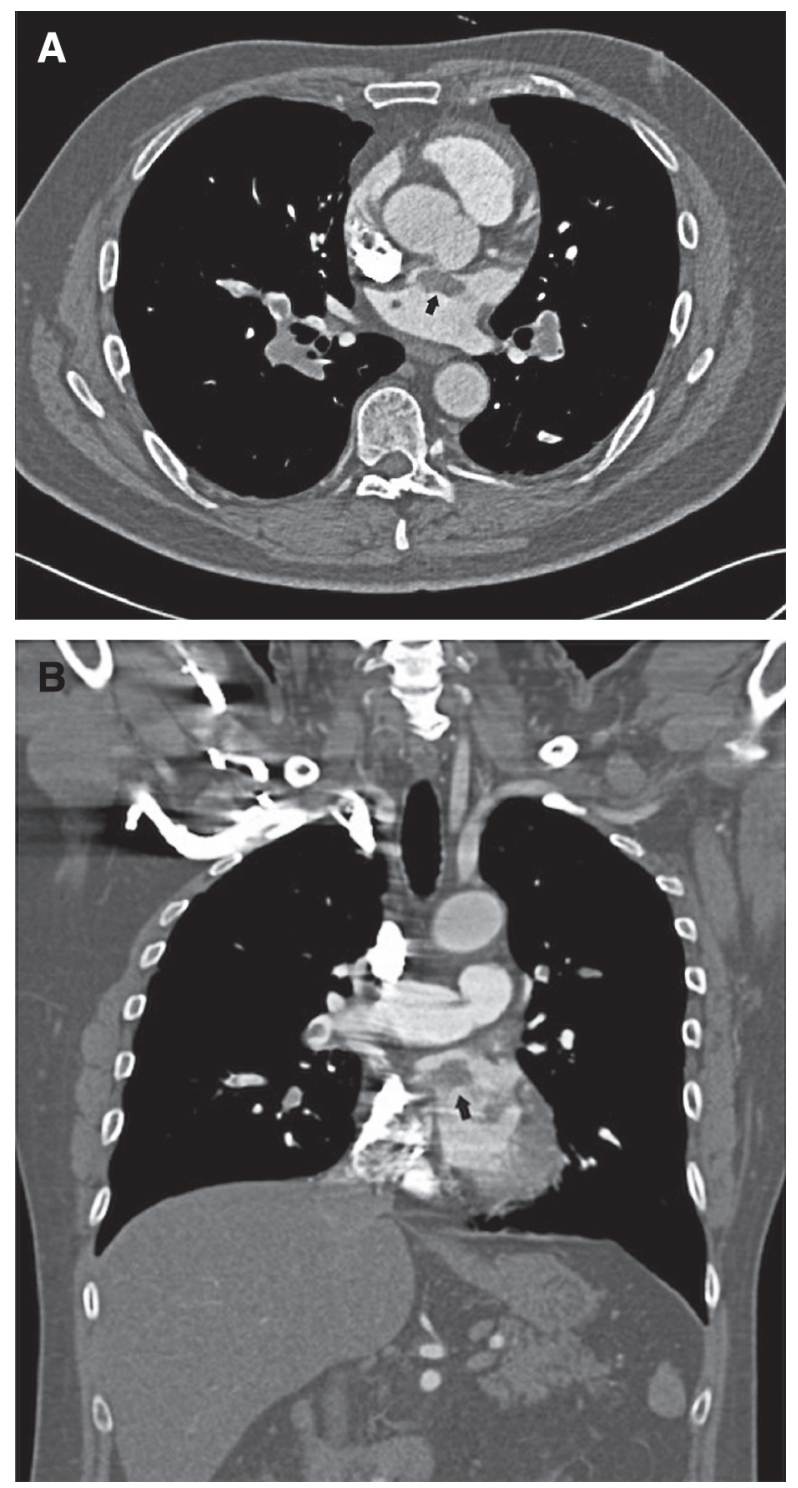

Fig. 4 The initial CT images taken on the same day, as depicted in Fig. 1, showing that the thrombus was not only in bilateral PAs but also in bilateral atria (arrows). (A) axial image and (B) coronal image. PAs: pulmonary arteries

\section{Discussion}

Acute PE causes widespread mortality and morbidity if not immediately addressed. Rapid diagnosis and treatment are essential, and CTPA offers a high diagnostic accuracy for PE. The modality demonstrates not only pulmonary thromboemboli but also right ventricular enlargement, indicating right ventricular dysfunction. ${ }^{1)} \mathrm{PFO}$, which is usually asymptomatic, is a common abnormality comprising a hole between the right and left atria, occurring in $15 \%-34 \%$ of the general population. ${ }^{2)}$ It is not usually investigated unless a patient has symptoms such as migraines or stroke.
In clinical practice, suspecting for and determining the presence of PFO is not easy in the emergency setting. Although the presence of PFO alone does not increase the risk of stroke, the prevalence of PFO is $40 \%-50 \%$ in patients who have cryptogenic stroke, especially those aged under 55 years. ${ }^{2}$ Its presence in patients with acute PE represents a risk factor for mortality. Several studies have demonstrated the increased risk of a recurrent ischemic stroke at the acute phase of PE with PFO, which were detected by TTE. ${ }^{5)}$ Furthermore, asymptomatic PE has been found in $10 \%-37 \%$ of patients with symptomatic ischemic stroke and PFO. ${ }^{\text {) }}$

A thrombus-in-transit is defined as a heart thrombus that is not attached to any intracardiac structure. When identified, thrombi-in-transit are commonly found to be associated with PE or paradoxical systemic embolism in the presence of an intracardiac shunt. A thrombus-in-transit via a PFO with impending paradoxical embolism carries a high mortality rate of approximately $30 \%{ }^{3,4)}$ This condition is associated not only with cryptogenic stroke but also with other ischemic complications (i.e., myocardial infarction or mesenteric ischemia). ${ }^{7)}$ Zhang et al. ${ }^{8)}$ demonstrated that the detection rate of PFO prevalence by CTPA corresponded to the known population prevalence, whereas TTE was less sensitive. Therefore, CTPA is recommended for the diagnosis of PFO with thrombus-in-transit in the acute setting.

The management strategy for a thrombus-in-transit has not yet been established because of the lack of randomized controlled trials or small case series. Although systemic thrombolysis and surgical embolectomy under cardiopulmonary bypass have shown a higher probability of survival than anticoagulant therapy for thrombus-in-transit without paradoxical embolization, they appear to be hazardous in patients with paradoxical embolization through PFO because they pose a risk of fragmentation or complete embolization. ${ }^{9-11)}$ Therefore, when identified, considering a case-by-case treatment approach by multidisciplinary teams is essential following a risk-benefit evaluation for preventing further life-threatening paradoxical embolization. In the present case, we performed mechanical thrombectomy as a first treatment of choice to prevent further neurological deterioration and administered both UFH and direct oral anticoagulants for PE and heart thrombus after performing mechanical thrombectomy.

Transcatheter PFO closure with the Amplatzer device was recently demonstrated to reduce the risk of recurrent ischemic stroke among patients aged under 60 years who 


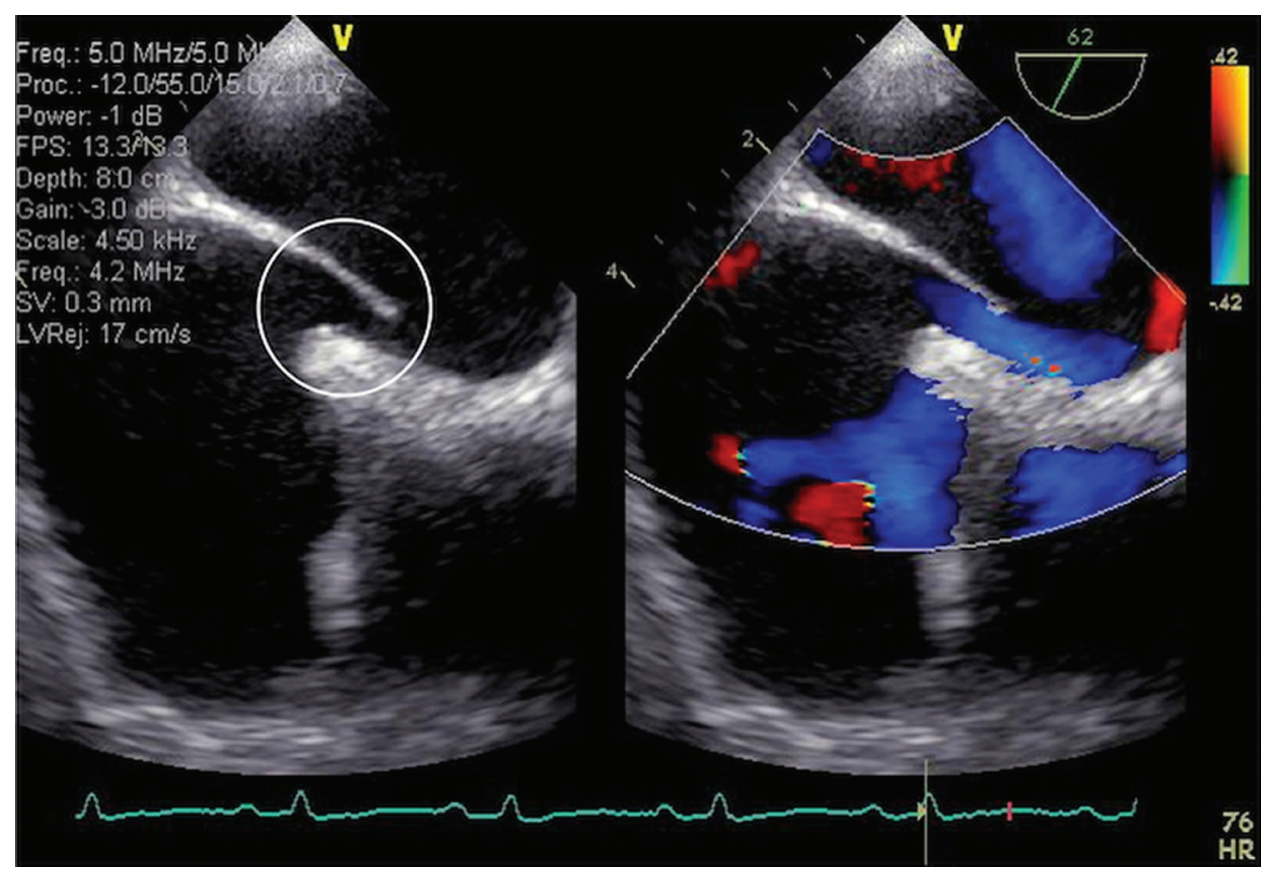

Fig. 5 TEE revealed a PFO (circle). PFO: patent foramen ovale; TEE: transesophageal echocardiography

had experienced a cryptogenic stroke, compared with medical therapy alone. ${ }^{12,13)}$ Another study demonstrated the efficacy of transcatheter PFO closure after achieving recanalization by mechanical thrombectomy in a young patient with cryptogenic stroke and PFO without PE. ${ }^{14)}$ Transcatheter PFO closure could be offered for patients at high risk of a recurrent embolic event, such as patients with atrial septal aneurysm, a long PFO tunnel, or a substantial right-to-left shunt. ${ }^{15)}$ Therefore, we recommend not only requiring rehabilitation for stroke but also considering PFO closure.

\section{Conclusion}

Not only cardiologists but also interventional neurologists should understand that a thrombus-in-transit via the PFO detected by CTPA provides a reliable indication for the sudden onset of an ischemic stroke in patients with symptomatic PE. When identified with paradoxical embolization, considering a case-by-case treatment approach by multidisciplinary teams is necessary to prevent further life-threatening paradoxical embolization.

\section{Acknowledgment}

The authors would like to thank Enago (www.enago.jp) for the English language review.

\section{Consent}

Prior written informed consent was obtained from the patient for the publication of this case report and its accompanying images.

\section{Disclosure Statement}

All authors declare no conflicts of interest.

\section{References}

1) Meinel FG, Nance JW, Schoepf UJ, et al: Predictive value of computed tomography in acute pulmonary embolism: systematic review and meta-analysis. Am J Med 2015; 128 : 747-759.e2.

2) American Heart Association: Patent foramen ovale (PFO). https://www.heart.org/en/health-topics/congenital-heartdefects/about-congenital-heart-defects/patent-foramenovale-pfo (Accessed: December 20, 2020).

3) Torbicki A, Galié N, Covezzoli A, et al: Right heart thrombi in pulmonary embolism: results from the International Cooperative Pulmonary Embolism Registry. J Am Coll Cardiol 2003; 41: 2245-2251.

4) Barrios D, Rosa-Salazar V, Morillo R, et al: Prognostic significance of right heart thrombi in patients with acute symptomatic pulmonary embolism: systematic review and meta-analysis. Chest 2017; 151: 409-416. 
5) Le Moigne E, Timsit S, Ben Salem D, et al: Patent foramen ovale and ischemic stroke in patients with pulmonary embolism: a prospective cohort study. Ann Intern Med 2019; 170: 756-763.

6) Lapergue B, Decroix JP, Evrard S, et al: Diagnostic yield of venous thrombosis and pulmonary embolism by combined CT venography and pulmonary angiography in patients with cryptogenic stroke and patent foramen ovale. Eur Neurol 2015; 74: 69-72.

7) Santangelo G, Ielasi A, Pattarino F, et al: Pulmonary embolism with migrating thrombus through patent foramen ovale: a case for a mixed pharmacological and percutaneous management. J Cardiol Cases 2019; 19: 19-21.

8) Zhang M, Tan S, Patel V, et al: Patent foramen ovale in patients with pulmonary embolism: A prognostic factor on CT pulmonary angiography? J Cardiovasc Comput Tomogr 2018; 12: 271-274.

9) Galeano-Valle F, Demelo-Rodríguez P, García-FernándezBravo I, et al: Early surgical treatment in patients with pulmonary embolism and thrombus-in-transit. $J$ Thorac Dis 2018; 10: 2338-2345.

10) Arboine-Aguirre L, Figueroa-Calderón E, Ramírez-Rivera A, et al: Thrombus in transit and submassive pulmonary thromboembolism succesfully treated with tenecteplase. Gac Med Mex 2017; 153: 129-133.

11) Athappan G, Sengodan P, Chacko P, et al: Comparative efficacy of different modalities for treatment of right heart thrombi in transit: a pooled analysis. Vasc Med 2015; 20: 131-138.

12) Saver JL, Carroll JD, Thaler DE, et al: Long-term outcomes of patent foramen ovale closure or medical therapy after stroke. N Engl J Med 2017; 377: 1022-1032.

13) Abdelaziz HK, Saad M, Abuomara HZ, et al: Long-term outcomes of patent foramen ovale closure or medical therapy after cryptogenic stroke: a meta-analysis of randomized trials. Catheter Cardiovasc Interv 2018; 92: 176-186.

14) Fu Q, Guo X, Mo D, et al: Young paradoxical stroke treated successfully with mechanical thrombectomy using solitaire and transcatheter closure of patent foramen oval. Int Heart $J$ 2017; 58: 812-815.

15) Chinese Collage of Cardiovascular Physicians: Recommendations from Chinese experts on management of patients with patent foramen ovale. Chin Heart J 2015, 373-379. 